https://helda.helsinki.fi

Spectral sensitivities of short- and long-wavelength sensitive cone mechanisms in the frog retina

Koskelainen, A.

Blackwell

1994

Acta Physiologica Scandinavica. 1994. 152: 115-124

http://hdl.handle.net/1975/948

Downloaded from Helda, University of Helsinki institutional repository.

This is an electronic reprint of the original article.

This reprint may differ from the original in pagination and typographic detail.

Please cite the original version. 


\title{
Spectral sensitivities of short- and long- wavelength sensitive cone mechanisms in the frog retina
}

\author{
A. KOSKELAINEN, ${ }^{1}$ S. HEMIL $\ddot{A}^{1}$ and K. DONNER ${ }^{2}$ \\ ${ }^{1}$ Laboratory of Physics, Helsinki University of Technology, FIN-02150 Espoo, Finland \\ and ${ }^{2}$ Department of Zoology, University of Helsinki, FIN-00100 Helsinki, Finland
}

\begin{abstract}
Koskelainen, A., Hemilä, S. \& Donner, K. 1994. Spectral sensitivities of short- and long-wavelength sensitive cone mechanisms in the frog retina. Acta Physiol Scand 152, 115-124. Received 25 October 1993, accepted 12 April 1994. ISSN 0001-6772. Laboratory of Physics, Helsinki University of Technology and Department of Zoology, University of Helsinki, Finland.
\end{abstract}

ERG mass photoreceptor responses were recorded across the isolated, aspartateperfused retina of the frog, Rana temporaria, in order to determine spectral sensitivities of cones. Cone responses were distinguished from rod responses by their faster kinetics, and responses from different cone types were isolated by selective background adaptation. Our main finding is that of a novel short-wavelength sensitive cone population peaking at about $431 \mathrm{~nm}$. Further, we find that the sensitivity spectrum of the dominant long-wavelength sensitive cone population fully accounts for the most common type of photopic ganglion cell spectrum. Both can be described by a nomogram with $\lambda_{\max }=562 \mathrm{~nm}$. This resolves a long-standing apparent conflict between cone absorbance spectra and ganglion cell sensitivities. Including the $502 \mathrm{~nm}$ cones previously described by microspectrophotometry, the frog possesses a collection of cones that could support trichromatic photopic vision.

Key pords: colour vision, cone photoreceptor, electroretinogram, frog, spectral sensitivity, visual pigment.

Just over half a century ago in this journal, Ragnar Granit published his summary of several years of work on the 'colour receptors of the frog's retina' (Granit 1942). This was the first demonstration in any species that single neurones (in his case ganglion cells) had different spectral sensitivities. For several decades the frog retina played a central role in the study of the physiological basis of colour vision, including pioneering electrophysiology (Bongard 1955, Bongard \& Smirnov 1957, Liberman 1957, Donner \& Rushton 1959), electron microscopy (Nilsson 1964a, b) and microspectrophotometry (Liebman \& Entine 1968). Colour opponency and colour coding as well as behavioural colour discrimination were extensively studied (reviewed by Orlov \& Kondrashov 1978; see also Maximov et al. 1985).

Thus one would expect the emerging picture of frog photoreceptor mechanisms to be more or less final. The consensus has been that there are four types of photoreceptors: two rods ('red' rhodopsin rods, $\lambda_{\max }=502 \mathrm{~nm}$ and 'green' rods, $\lambda_{\max }=433 \mathrm{~nm}$ ) and two cones. According to Liebman \& Entine (1968), single cones and principal members of double cones have $\lambda_{\max }=$ $575 \mathrm{~nm}$, while accessory members of double cones contain a green pigment described by the authors as spectrally indistinguishable from rhodopsin.

It therefore came as a considerable surprise when, during ERG recordings with quite different objectives, we observed a 'paradoxical' cone sensitivity in the blue. In view of other unresolved problems, particularly the fact that the most common type of photopic ganglion cell sensitivity cannot be synthesized from the absorbance spectra of Liebman \& Entine (1968) (see Reuter 1969), we decided to reinvestigate frog cone mechanisms globally, using ERG 
recording across the isolated aspartatesuperfused retina. This technique has the advantage that it picks up the responses of every substantively represented photoreceptor type, obviating the sampling problems connected both with microspectrophotometry (MSP) and ganglion cell recordings. The recorded signal sums the responses of thousands of cells and hence has a comparatively high signal/noise ratio. The main drawback is that it pools contributions from all cells that respond to the stimulus, so isolation of a single photoreceptor type requires careful analysis (see Methods).

Our main new result is the characterization of a short-wavelength sensitive cone mechanism $\left(\lambda_{\max }=431 \pm 3 \mathrm{~nm}\right)$, not described before in anuran amphibians. Further, we present a new determination of the spectrum of the longwavelength sensitive cones, where fitting standard nomograms gives $\lambda_{\max }=562 \pm 3 \mathrm{~nm}$ rather than the usually cited $570-580 \mathrm{~nm}$. The spectrum of the rhodopsin rods $\left(\lambda_{\max }=501 \mathrm{~nm}\right)$ was measured as a methodological control. Somewhat disappointingly we were unable to isolate reliably a signal from the 'rhodopsin-like' cones described by Liebman \& Entine (1968). Accepting the MSP evidence for these, however, the frog retina must now be considered to have three spectrally different cone types (in addition to two rod types). The functions of this set of photoreceptors cannot be fully understood on the basis of current notions on (dichromatic) frog colour vision.

\section{METHODS}

Animals, perfusion and recording. Common frogs (Rana temporaria) were caught in the wild in Leningrad Region (Russia) in late autumn. They were maintained under hibernation conditions in neardarkness at $4{ }^{\circ} \mathrm{C}$ without feeding. Before an experiment, a frog was warmed overnight in darkness to ca $15^{\circ} \mathrm{C}$. It was double-pithed, the eyes were excised, and one retina was isolated in cool Ringer solution in dim red light. The retina was mounted in a specimen holder where the vitreal side rested on a plexiglass hemisphere and the photoreceptor (upper) side was continuously perfused (see Donner et al. 1988). The perfusing Ringer contained (in $\mathrm{mm}$ ) $\mathrm{NaCl}, 95 ; \mathrm{KCl}$, 3 ; $\mathrm{CaCl}_{2}, 0.9 ; \mathrm{MgCl}_{2}, 0.5$; glucose, 10 ; HEPES $\mathrm{pH}$ buffer, $6 ; \mathrm{NaHCO}_{3}$ buffer, 6 ; and Na-aspartate, 2 , to block synaptic transmission from the photoreceptors. To this was added $5 \%$ of Leibovitz culture medium L-15 (Sigma) to improve the viability of the retina.
$\mathrm{pH}$ was adjusted to 7.5 with $\mathrm{HCl}$. Temperature was $\mathrm{ca}$ $12{ }^{\circ} \mathrm{C}$. Photoresponses were DC-recorded with $\mathrm{Ag} / \mathrm{AgCl}$ electrodes as transretinal voltages, eightpole analogue-filtered at $100 \mathrm{~Hz}$, digitized (sampling rate of $200 \mathrm{~s}^{-1}$ ) and stored on disc.

Stimulus lights. A halogen lamp driven by a stabilized current source provided the light for both the stimulus and the background channel. The light intensities in the two channels were independently regulated by neutral density filters and wedges. Stimuli were $20 \mathrm{~ms}$ full-field light flashes of different wavelengths. Fifteen Schott DIL and six Schott IL interference filters provided nearly monochromatic lights suitably spaced over the range $396-681 \mathrm{~nm}$. The transmission of the filters was measured with a Beckman spectrophotometer. DIL filters had halfwidths about $10 \mathrm{~nm}$ and peak transmissions $11-19 \%$ except for those at 660 and $681 \mathrm{~nm}$ which had broader bands $(20$ and $26 \mathrm{~nm})$ and lower peak transmissions (about 5\%). IL-filters had half-widths of 11-17 nm and maximum transmissions of 32-57\%. Background lights were delivered through Schott edge filters OG 530 or OG 570 (sharp edge transmitting above 530 and $570 \mathrm{~nm}$, respectively) or a Schott IL $430 \mathrm{~nm}$ interference filter. These were our standard backgrounds for selective adaptation and will be referred to as 'orange', 'red' and 'blue', respectively.

The calibration of stimulus lights was based on an Airam UVM-8 radiometer. The factory's spectral calibration of this device had been carefully checked and corrected against a vacuum thermo-element (Spindler \& Hoyer). A silicon pin diode (HUV$1000 \mathrm{~B}, \mathrm{EG} \& \mathrm{G}$ electro-optics) was then calibrated against the Airam, which was too large to fit into the place of the retina. With each of the interference filters in turn interposed in the light beam, the transmitted photon flux (photons $\mathrm{m}^{-2} \mathrm{~s}^{-1}$ ) was first measured with the Airam meter. Then the pin diode was placed in the same position and its photovoltage read, giving its quantal sensitivity $\left[\mathrm{V} /\left(\right.\right.$ photons $\left.\left.^{-2} \mathrm{~s}^{-1}\right)\right]$ at each wavelength. The actual stimulus lights were measured with the pin diode in the place of the retina in the specimen chamber.

Determination of spectral sensitivities. The isolation of distinct spectral mechanisms basically followed the logic of Stiles' (1949) 'two-colour method', where stimulus flashes of different wavelengths are superimposed upon steady backgrounds of wavelengths chosen to isolate (ideally) one mechanism by suppressing all others. Spectral sensitivities are obtained as reciprocals of criterion threshold intensities at the different stimulus wavelengths. If only one receptor mechanism is active, an adapting background does not change the shape of the sensitivity spectrum, only the overall level. When photoreceptor responses are recorded, two additional parameters are available for analysis: response waveform, used to differentiate between cones and rods, and the satu- 
rating response amplitude at different wavelengths, used to identify mixed responses originating in spectrally different photoreceptors. It is assumed that when a photon elicits an excitation in the photoreceptor, the size and shape of the response is independent of the wavelength of the photon [the 'univariance principle' of Naka \& Rushton (1966)]. If only one type is active, the intensity-response curve (in a $\log -\log$ plot) will then retain both its shape and its position on the log response axis (the saturating response amplitude remains constant) and only move along the log intensity axis as stimulus wavelength is changed. The position on the log intensity axis can be defined by a 'threshold' intensity $I_{\mathrm{t}}$ corresponding to any (small) response criterion. Spectral sensitivity, which measures the probability that a photon of a certain wavelength shall elicit an excitation in the photoreceptor, is inversely proportional to the threshold intensity expressed as quantum flux incident on the retina (photons $\mathrm{m}^{-2} \mathrm{~s}^{-1}$ ).

Photoreceptor terminology. We shall call longwavelength sensitive cones 'red', middle-wavelengthsensitive cones 'green' and short-wavelength sensitive cones 'blue'. We shall refer to the (green-sensitive) 'red rods' as rhodopsin rods, and to the 'green rods' as blue-sensitive rods or $433 \mathrm{~nm}$ rods.

Curves fitted to the spectra. Standardized template curves are used for unified description and interpolation and for comparing sensitivity spectra with pigment absorbance spectra. For cones, we use the ad hoc sixth-order polynomial expression applied by Baylor et al. (1987) to the spectral sensitivities of macaque cones, also found to fit salamander cones (Perry \& McNaughton 1991). It incorporates the principle of shape-invariance on a relative wave number $\left(\lambda_{\max } / \lambda\right)$ scale (Mansfield 1985, MacNichol 1986):

$\log S=\sum a_{\mathrm{n}}\left[\log _{10}\left(\lambda_{\max } / \lambda \lambda_{\mathrm{r}}\right)\right]^{\mathrm{n}}$,

where $\lambda_{\mathrm{r}}=561 \mathrm{~nm}$ and $\lambda$ is in units of $\mu \mathrm{m}$. The coefficients $\left(a_{0}-a_{6}\right)$ are $-5.2734,-87.403,1228.4$, $-3346.3,-5070.3,30881,-31607$. We refer to this as the 'cone nomogram'. The iodopsin absorption spectrum of Wald et al. (1955) in its polynomial formulation by Dawis (1981) fitted the spectral sensitivities of red cones about equally well as Eqn (1), but was clearly inferior for blue cones.

The rod sensitivity spectrum was fitted by a template based on recent rhodopsin absorbance measurements by Partridge \& de Grip (1991). Their data are from bovine rhodopsin, so the template was shifted for best fit to the frog pigment, observing the $\lambda_{\max } / \lambda$ rule (see above). The fit was clearly superior to that of the Dartnall (1953) nomogram, which drops too shallowly in the long-wavelength end. [When fitting the Dartnall nomogram, we used the polynomial expression of Dawis (1981).]
Rhodopsin self-screening. If the probability that a photon absorption sets off the transduction cascade is independent of wavelength, the relative thresholds $I_{\mathrm{t}}(\lambda) / I_{\mathrm{t}}\left(\lambda_{\max }\right)$ at different wavelengths $\lambda$ are in principle equal to the relative extinction values $\epsilon(\lambda) / \epsilon\left(\lambda_{\max }\right)$ of the photopigment. In rods with their long outer segments, however, self-screening of rhodopsin must be taken into account, and instead of $\epsilon(\lambda) / \epsilon\left(\lambda_{\max }\right)$, we used the expression

$\frac{1-10^{-0.5 \epsilon(\lambda) / \epsilon\left(\lambda_{\max }\right)}}{1-10^{-0.5}}$

corresponding to optical density 0.5 at peak wavelength (Reuter 1969). In frog cones with their very small outer segments (Saxén 1954; Nilsson 1964a), self-screening is negligible.

Isolation of the different receptor types. The sensitivity spectrum of the rhodopsin rods was determined under a very dim blue background light, close to complete dark-adaptation. A strong (unattenuated) blue background was used to isolate responses of the red cones. The blue cones were studied under unattenuated orange background, which strongly depressed rhodopsin rods and red cones. Because photoresponses from strongly light-adapted rods are quite fast (Donner $e t$ al. personal communication) and could possibly contribute to the peak amplitude of 'blue-cone' responses, we checked for, and if necessary eliminated, interference from rods $(433 \mathrm{~nm}$ rods and residual responses from rhodopsin rods) by a 'doubleflash' technique. This technique takes advantage of the fact that the recovery of strongly saturated rod responses is always slow. Figure 1 a shows how a $1 \mathrm{~s}$ pre-exposure with unattenuated blue light elicited saturating responses in rods, as well as large cone respones. Upon termination of this conditioning stimulus, cones recovered almost immediately, but rods remained saturated for several seconds. Thus a test flash delivered $2 \mathrm{~s}$ later elicited a pure cone response. The waveform was purified by subtracting the response to the conditioning flash alone. The traces in Figure $1 \mathrm{~b}$ show such resultant responses to flashes of unattenuated $519 \mathrm{~nm}$ light delivered after 'blue' conditioning exposures of three different intensities: unattenuated and attenuated by 1 and 3 $\log$ units, respectively. In the test response after the weakest pre-exposure, a long saturated rod tail is still evident, whereas the test responses after the two stronger conditioning exposures lack this tail and are virtually identical. Thus both the latter pre-exposures suppressed rods without depressing the saturated response of cones.

In practice, the unattenuated orange and blue backgrounds turned out to isolate cones well enough, and the double-flash technique mainly served to confirm that our presumed cone sensitivities were not distorted by rods. We also used it for eliminating any 
remaining rod component while (unsuccessfully) attempting to find a green-cone contribution in saturated cone responses (such as those shown in Fig. 1).

Screening of cones by rhodopsin insignificant. The measured cone spectra might in principle be affected by rhodopsin screening. We therefore needed to know the density of unbleached rhodopsin under the unattenuated red, orange and blue background lights. Rhodopsin bleaching velocities were estimated to $34 \% \min ^{-1}, 2.5 \% \mathrm{~s}^{-1}$ and $1 \% \mathrm{~min}^{-1}$, respectively (accuracy $\pm 30 \%$ ). Thus, under the red and orange backgrounds, the rhodopsin would be completely bleached. The photoproducts, on the other hand, do not absorb significantly in the spectral regions with which we are concerned here. Under the blue background, screening by rhodopsin could cause some underestimation of sensitivities in the green, and screening could be significant if spectra are determined without steady background light. Indeed, a clear sensitivity reduction around $500 \mathrm{~nm}$ was observed in red-cone spectra determined by the double-flash technique in two dark-adapted retinas (see below).

\section{RESULTS}

\section{The sensitivity spectrum of rhodopsin rods}

The much-studied spectrum of the rhodopsin rods was recorded as a methodological control. Mean relative sensitivities from experiments on three retinas are shown in Figure 2. The fulldrawn curve is based on the accurate spectrophotometric recordings of (bovine) rhodopsin by Partridge \& de Grip (1991). The curve based on their tabulated absorbance values has been adjusted for self-screening and fitted observing the $\lambda_{\max } / \lambda$ rule (see Methods). The best fit was obtained with $\lambda_{\max }=501 \mathrm{~nm}$, well within the interval $500.7<\lambda_{\max }<501.4$ given by Partridge \& de Grip for the absorbance of $R$. temporaria rhodopsin. The curve describes the data well over the whole range where it is valid. We have truncated it at $625 \mathrm{~nm}$, where spectrophotometry becomes unreliable due to very low absorbances. (A conventional extrapolation would be by a falling exponential, a sloping straight line on the logarithmic ordinates of Fig.

2.) The conclusion is that the ERG sensitivity spectrum we measure closely reproduces the photopigment absorbance spectrum.

The crosses in Figure 2 plot spectral sensitivities of dark-adapted ganglion cells obtained by threshold determinations in the eyecup preparation (Reuter 1969). The excellent agreement with the ERG data at most wavelengths indicates that the direction of light incidence or other preparation-related peculiarities did not significantly affect the results. In the far red, however, ganglion cells appear to be relatively more sensitive. The discrepancy here almost certainly arises from a red-cone contribution to the ganglion cell thresholds (which were recorded with step stimuli, whereby the rod/cone difference in response kinetics is of little importance). In our ERG recordings, dim-flash responses of darkadapted rods and cones were easily distinguished, as times-to-peak differed approximately by a factor of 10 .

\section{Red cones}

Photoresponse maveform. Figure 3 shows a family of cone responses to red flashes increasing in $0.5 \mathrm{log}$ unit steps, recorded under strong blue background. As shown before, the waveforms of dim-flash ERG responses under these conditions closely resemble current responses of single cones (Perry \& McNaughton 1991, Koskelainen et al. 1993). Thus it is likely that small responses, on which the spectral sensitivity determinations depend, represent almost pure cone activity. By contrast, the larger responses have a protracted tail that suggests rod intrusion.

Spectral sensitivities. Spectral sensitivities of red cones were recorded in six retinas: in four against strong blue background and in two by the double-flash technique without background. Mean relative sensitivities from the four 'background' spectra are shown in Figure 4, together with the cone nomogram (Eqn 1) for $\lambda_{\max }=$ $562 \mathrm{~nm}$, which provided the best fit to the data. The same $\lambda_{\max }$ was obtained when the nomogram was fitted to the long-wavelength end of the 'double-flash' spectra. (In the latter case, sensitivities at shorter wavelengths were neglected, as they seemed to be depressed by rhodopsin screening: see Methods.) Though the fit of the nomogram is generally fair, it is worth noting that recorded sensitivities in the far red fell more steeply than predicted.

Liebman \& Entine (1968), on the basis of MSP recordings in $R$. pipiens, gave $\lambda_{\max }$ as $575 \mathrm{~nm}$ for the red cones. To check for a possible species difference, we recorded red-cone spectra in two specimens of $R$. pipiens, but the results $\left(\lambda_{\max } \approx 562-563 \mathrm{~nm}\right)$ gave no indication of at 
(a)
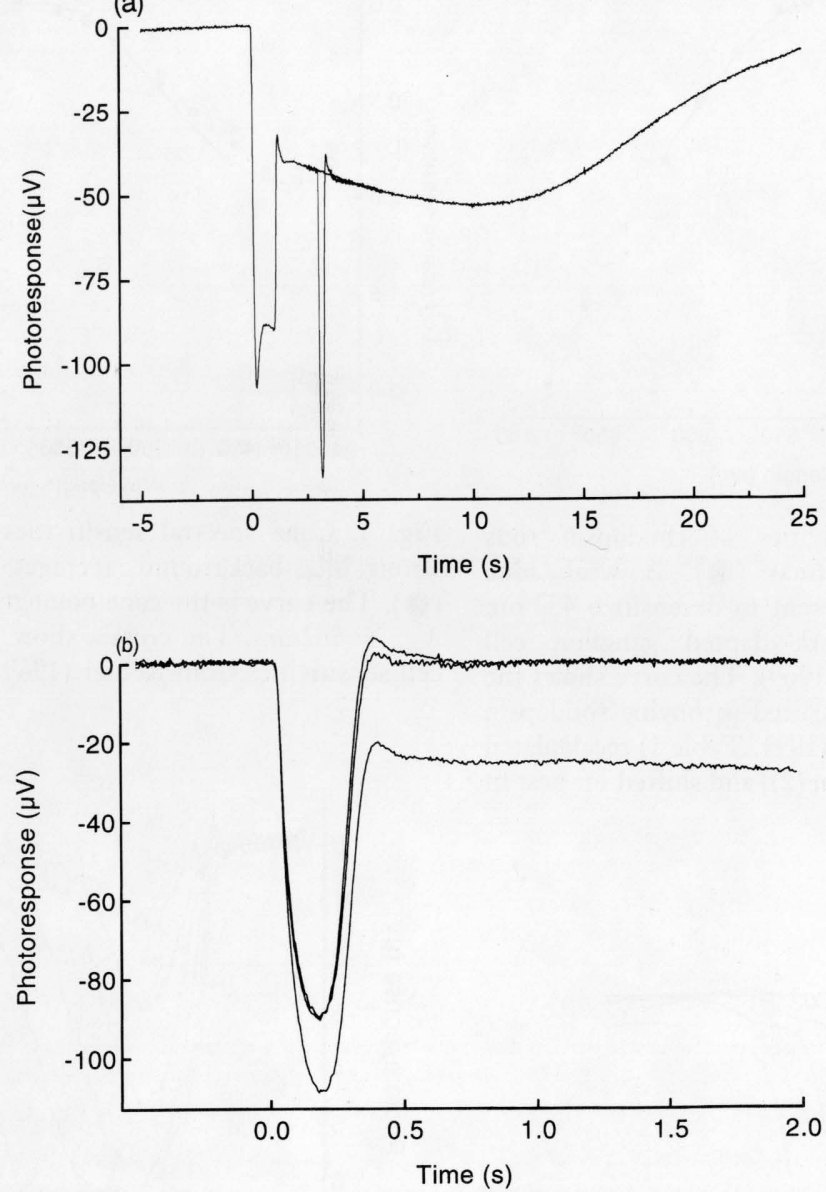

Fig. 1. Isolation of cone responses by the double-flash technique illustrating the slow recovery of strongly saturated rod responses and the fast kinetics of cone responses. (a) Combination of the response to conditioning stimulus alone (unattenuated blue light, duration $1 \mathrm{~s}$ ) and, superposed on this, the response to the test flash (at $3 \mathrm{~s}$ in the figure: $20 \mathrm{~ms}$ flash of unattenuated $519 \mathrm{~nm}$ light) recorded in a separate run after an identical pre-exposure. The conditioning stimulus delivered at time 0 elicits a large mixed response of rods and cones. At $\mathrm{OFF}$, the cone response rapidly returns to baseline, but the rod response remains saturated for several seconds; thus the test flash delivered in this interval elicits a pure cone response. Rod saturation appears not as a plateau but as a slow rise for more than $10 \mathrm{~s}$ because of glial currents evoked by the rod response. (b) Three test responses to identical flashes $(20 \mathrm{~ms}$ unattenuated $519 \mathrm{~nm}$ light) recorded after different preexposures: $1 \mathrm{~s}$ blue conditioning stimulus either attenuated by $3 \log$ units (lowermost response), by $1 \log$ unit, or unattenuated. The test responses after the two latter pre-exposures coincide over most of their course, indicating, firstly, that no rod contribution remained and secondly, that the cones had fully recovered. (The response after unattenuated conditioning light is identical to that shown in (a) and can be distinguished by a slightly larger overshoot).

least a systematic difference compared with $R$. temporaria. The discrepancy between our results and those of Liebman \& Entine (1968) is considered in detail in the Discussion.

\section{Blue cones}

Under the strong orange background, even unattenuated yellow flashes elicited no recordable 


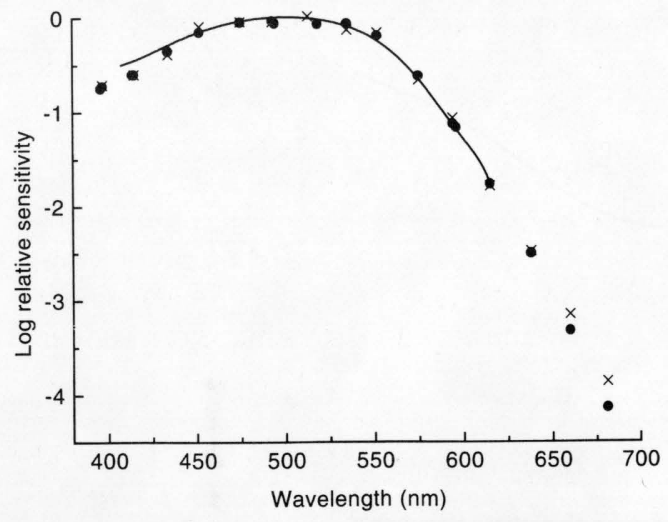

Fig. 2. Spectral sensitivities of rhodopsin rods averaged from three retinas (-). A weak blue background light was present to desensitize $433 \mathrm{~nm}$ rods. Crosses show dark-adapted ganglion cell sensitivities from Reuter (1969). The curve shows the absorbance spectrum measured in bovine rhodopsin by Partridge and de Grip (1991, Table 1) recalculated for optical density 0.5 [Eqn (2)] and shifted for best fit at $\lambda_{\max }=501 \mathrm{~nm}$.

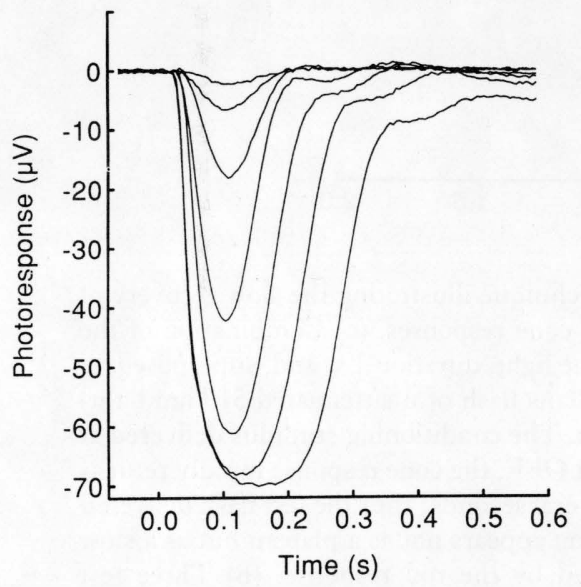

Fig. 3. Response family of red cones (red flashes against strong blue background), flash intensities increa.ing in $0.5 \mathrm{log}$ unit steps. Each trace is an average of four single responses.

responses, indicating that rhodopsin rods and red cones were saturated. Blue flashes, however, evoked photoresponses with cone-like kinetics as shown in Figure 5. The double-flash technique allowed us definitely to rule out the $433 \mathrm{~nm}$ rods. This indicates the presence of a functional population of short-wavelength sensitive cones.

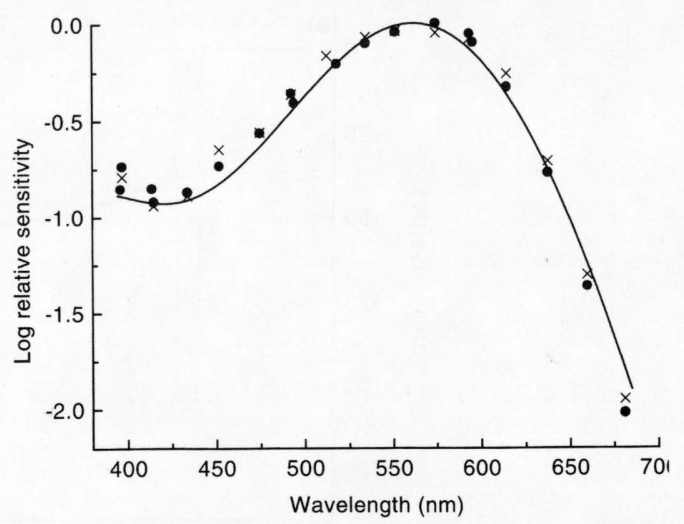

Fig. 4. Cone spectral sensitivities recorded against strong blue background, averages from four retinas (O). The curve is the cone nomogram [Eqn (1)] with $\lambda_{\max }=562 \mathrm{~nm}$. The crosses show photopic ganglion cell sensitivities from Reuter (1969).

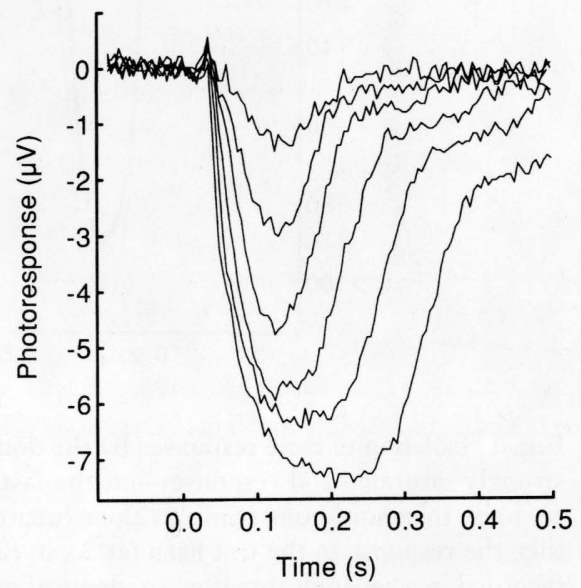

Fig. 5. Response family of the blue-sensitive cone mechanism (blue flashes against strong orange background) illustrating the similarity in waveforms to red-cone responses (Fig. 3). Flash intensities increase in $0.4 \mathrm{log}$ unit steps. Each trace is an average of eight single responses.

Clearly, response waveforms are similar to those of red cones (Fig. 3), but they differ from those of dark-adapted blue cones in salamander, which return rather slowly towards baseline after bright flashes (Perry \& McNaughton 1991). When comparing kinetics it should be borne in mind, though, that the blue-cone responses in Figure 5 may be somewhat light-adapted and the red-cone responses in Figure 3 are significantly 


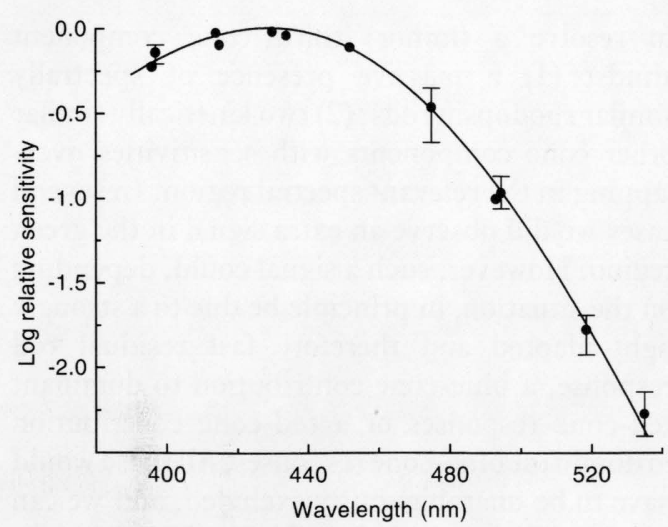

Fig. 6. Cone spectral sensitivities recorded against strong orange background, averages from four retinas. The curve is the cone nomogram for $\lambda_{\max }=431 \mathrm{~nm}$. Bars show representative ranges of variation at some wavelengths.

so. The saturating amplitude of the blue-sensitive response in our experimental conditions was typically $10 \%$ or less of that of dark-adapted red cones. (Dark-adapted red-cone responses were obtained by recording responses to rod-matched $642 \mathrm{~nm}$ and $519 \mathrm{~nm}$ flashes in the dark-adapted retina and subtracting the latter from the former.) This suggests that red cones outnumber blue cones by roughly one order of magnitude.

Spectral sensitivities of blue cones were recorded in four retinas under orange background. The cone nomogram with $\lambda_{\max }=$ $431 \mathrm{~nm}$ provides a good fit to the averaged data (Fig. 6).

\section{DISCUSSION}

\section{Blue cones}

This is the first demonstration of a functional population of short-wavelength sensitive cones in anuran amphibians. Admittedly, Hárosi in his Taniguchi lecture (1982) mentions having seen four $R$. catesbeiana cones with blue-absorbing visual pigment (out of 38 recorded by MSP), but he presents no actual data. Liebman \& Entine (1968) found no blue cones, although such were clearly present in both the $R$. pipiens retinas studied by us. Salamanders possess both blue and UV-cones (Perry \& McNaughton 1991), but we found no evidence for the latter type in frogs.
The fact that blue cones have escaped physiological detection so effectively may provide a clue to their function. In ganglion cell recordings, blue-cone-driven responses would be hard to distinguish from responses driven by the spectrally close $433 \mathrm{~nm}$ rods on the condition that they are similarly connected to the ganglion cell, i.e. in the manner of an antagonistic receptive-field surround (Donner \& Grönholm 1984). Indeed, in cone-driven centre-type responses the relative sensitivity in the blue is generally modest and could be due to the $\beta$-band of the red-cone pigment (Reuter 1969). Only in a few cases has it seemed possible to depress selectively the short-wavelength sensitivity of the receptive-field centre mechanism by backgrounds, so as to suggest independent input from a 'blue' receptor into the centre (Fig. 4 in Donner 1959; see also Fig. 2 in Bäckström \& Reuter 1975).

\section{Red cones}

The red-cone spectrum recorded here accounts well for the most common type of photopic ganglion cell sensitivities, as evident from the good agreement between circles and crosses in Figure 4. This resolves a troublesome discrepancy between the MSP of Liebman \& Entine (1968) and the ganglion cell sensitivities recorded by Granit (1942) and Reuter (1969). We found no fixed species difference between $R$. pipiens and $R$. temporaria. Thus our results conflict with those of Liebman \& Entine, although less so than suggested by the large $\lambda_{\max }$ difference ( 562 vs. $575 \mathrm{~nm})$ :

(1) A simple comparison of $\lambda_{\max }$ values is really relevant only if spectra can be described by a common template. Even observing a $\lambda_{\max } / \lambda$ transformation (Mansfield 1985, MacNichol 1986), this appears to be no more than an approximate idealization. The cone nomogram is not a good fit to the long-wavelength limb of our red-cone spectral sensitivities (Fig. 4), and even less so to the MSP data of Liebman \& Entine (1968, their Fig. 16). We have preferred to use a conventional curve rather than an arbitrary construct, but it would be easy to devise a template fitting our data best when positioned at $\lambda_{\max }=575 \mathrm{~nm}$. Thuse we do not claim that $562 \mathrm{~nm}$ is the 'true' $\lambda_{\max }$ of frog red cones. Our central result is that red cone and ganglion cell 
sensitivities agree well (contrary to what has been believed), and when described with the Baylor et al. (1987) nomogram, both are best fit when the parameter $\lambda_{\max }$ (Eqn 1) is set equal to $562 \mathrm{~nm}$.

(2) It is difficult to assess how large the discrepancy in actual experimental data is, because the presentation of Liebman \& Entine (1968) is confused. The averaged red-cone absorbance spectrum they show (Fig. 16) peaks at $c a 587 \mathrm{~nm}$, far from the $575 \mathrm{~nm}$ given in the text. If we assume this to be a simple mistake of axis labelling, and shift their average curve to $575 \mathrm{~nm}$, only a minor 'hump' of extra sensitivity around $600 \mathrm{~nm}$ remains compared with our data (followed by a steep drop in the low-absorbance range beyond $625 \mathrm{~nm}$, which is clearly an artifact).

(3) Nevertheless, we can think of at least one mechanism that might really produce variable amounts of extra long-wavelength sensitivity. Adult bull-frogs ( $R$. catesbeiana) are known to retain vitamin- $\mathrm{A}_{2}$-based pigment in their retina in a dorso-ventral gradient, varying with season and other environmental factors (Reuter $\mathrm{et} \mathrm{al}$. 1971). Could this (to a smaller extent) be the case in some other frog species, too? Liebman \& Entine (1968) mention this possibility, having seen several $R$. pipiens with $\operatorname{rod} \lambda_{\max }$ as high as $507 \mathrm{~nm}$. In the same species, the early receptor potential (ERP) recordings of Goldstein (1968) indicate red-cone $\lambda_{\max }$ as high as $580 \mathrm{~nm}$, while the mass cone sensitivities of Hood \& Mansfield (1972) are in fact consistent with a nomogram peaking at ca $565 \mathrm{~nm}$. Bongard (1955) and Bongard \& Smirnov (1957) placed the red-cone sensitivity peak in $R$. ridibunda at $570-580 \mathrm{~nm}$ (based on ganglion cell recordings), while Granit $\&$ Wrede (1937) found $\lambda_{\max } \approx 560 \mathrm{~nm}$ in the closely related $R$. esculenta (ERG recordings). These results suggest considerable biological variability.

\section{The silence of the green cones}

Our failure to isolate a response component from 'rhodopsin-like' cones merits comment. It would be surprising if this cone type were missing in $R$. temporaria, being present not only in $R$. pipiens but apparently also in $R$. catesbeiana (Liebman \& Entine 1968, Hárosi 1982). It is more likely that it was beyond the capacity of our ERG technique to resolve a (minor) third cone component amidst (1) a massive presence of spectrally similar rhodopsin rods; (2) two kinetically similar other cone components with sensitivities overlapping in the relevant spectral region. In several cases we did observe an extra signal in the green region. However, such a signal could, depending on the situation, in principle be due to a strongly light-adapted and therefore fast residual rod response, a blue-cone contribution to dominant red-cone responses or a red-cone contribution to dominant blue-cone responses. All these would have to be unambiguously excluded, and we can only say that we were unable to find the right conditions.

\section{Functional considerations}

Colour-opponent ganglion cells: rod-cone shift in both centre and surround? Bäckström \& Reuter (1974) pointed out that antagonism between a receptive field centre mechanism driven by rhodopsin rods and a surround mechanism driven by $433 \mathrm{~nm}$ rods could provide frogs with scotopic colour discrimination. Selective adaptation with yellow backgrounds creates a situation where red cones take over in the centre, but $433 \mathrm{~nm}$ rods apparently continue to drive the antagonistic surround (Reuter 1969, Reuter \& Virtanen 1972, Bäckström \& Reuter 1975, Donner \& Grönholm 1984). The blue cones could obviously subserve a third type of colour opponency (red vs. blue cones) at still more light-adapted levels.

Granit's modulators. The dominator/modulator theory of Granit $(1942,1943)$ is of purely historical interest today, but there is no reason to doubt his experimental results showing narrow ('modulator') spectral sensitivities in ganglion cells under certain conditions. Although they may be dismissed as accidental results of interactions between variably adapted photoreceptors (Bongard \& Smirnov 1957), such sharp spectral structures, recordable only in a small subset of ganglion cells (cf. Donner \& Grönholm 1984), could well be functionally significant. Three cone types would at least provide sufficient degrees of freedom to generate Granit's 'photopic modulators'. It is noteworthy that little physiology or behaviour has subsequently been done at the strongly light-adapted levels 
where Granit was working (long adapting exposures to bright sunlight). This might be one reason why, more than $50 \mathrm{yr}$ later, the frog's use of spectral information can still not be considered as fully understood.

We wish to thank Mr Tuomo Lerber and Mr Antti Miettinen for skilful technical assistance and $\mathrm{Dr}$ Victor I. Govardovskii for providing us with frogs, which were not obtainable in Finland due to a warm autumn followed by sudden onset of winter. We are indebted to Professor Kai Otto Donner for important help with the spectral calibrations. We also thank Professor Tom Reuter for valuable comments on the manuscript. This work was supported by the Academy of Finland (grant 01/1011872).

\section{REFERENCES}

BäCKSTRÖM, A.-C. \& ReUTeR, T. 1974. Opponent colour interaction between two kinds of rod signals in the frog's retina. Physica Norvegica 7, 187-189.

BäскSTRÖM, A.-C. \& ReUTER, T. 1975. Receptive field organization of ganglion cells in the frog retina: contributions from cones, green rods and red rods. 7 Physiol 246, 79-107.

Baylor, D.A., Nunn, B.J. \& Schnapf, J.L. 1987. Spectral sensitivity of cones of the monkey Macaca fascicularis. 7 Physiol (Lond) 390, 145-160.

BONGARD, M.M. 1955. Colorimetry in animals. Dokl Akad Nauk SSSR 103, 239-242 (in Russian).

Bongard, M.M. \& Smirnov, M.S. 1957. Spectral sensitivity curves for receptors connected to single fibers of the optic nerve of the frog. Biofizika 2, 336-342 (in Russian).

DarTNALL, H.J.A. 1953. The interpretation of spectral sensitivity curves. Brit Med Bull 9, 24-30.

DAwIS, S.M. 1981. Polynomial expressions of pigment nomograms. Vision Res 21, 1427-1430.

DonNer, K. \& GröNhOLM, M.-L. 1984. Center and surround excitation in the receptive fields of frog retinal ganglion cells. Vision Res 24, 1807-1819.

Donner, K., Hemilä, S. \& Koskelainen, A. 1988. Temperature-dependence of rod photoresponses from the aspartate-treated retina of the frog (Rana temporaria). Acta Physiol Scand 134, 535-541.

DoNNER, K.O. 1959. The effect of a coloured adapting field on the spectral sensitivity of frog retinal elements. I Physiol (Lond) 149, 318-326.

DonNeR, K.O. \& Rushton, W.A.H. 1959. Retinal stimulation by light substitution. 7 Physiol 149, 288-302.

GoLDSTEIN, E.B. 1968. Visual pigments and the early receptor potential of the isolated frog retina. Vision Res 8, 953-963.
Granit, R. 1942. Colour receptors of the frog's retina. Acta Physiol Scand 3, 137-151.

GRANit, R. 1943. A physiological theory of colour perception. Nature 151, 11-14.

Granit, R. \& Wrede, C.M. 1937. The electrical responses of light-adapted frogs' eyes to monochromatic stimuli. 7 Physiol 89, 239-256.

Hárosi, F.I. 1982. Recent results from single-cell microspectrophotometry: cone pigments in frog, fish, and monkey. Color Res Appl 7, 135-141.

Hood, D.C. \& Mansfield, A.F. 1972. The isolated receptor potential of the frog isolated retina: action spectra before and after extensive bleaching. Vision Res 12, 2109-2119.

Koskelainen, A., Donner, K., Lerber, T. \& Hemilä, S. 1993. $\mathrm{pH}$ regulation in frog cones studied by mass receptor photoresponses from the isolated retina. Vision Res 33, 2181-2188.

Liberman, E.A. 1957. The nature of the information transmitted to the brain by a single nerve fiber from two receptor types in the frog retina. Biofizika 2, 427-430 (in Russian).

Liebman, P.A. \& Entine, G. 1968. Visual pigments of frog and tadpole (Rana pipiens). Vision Res 8, $761-775$.

MACNiCHOL, E.F. JR. 1986. A unifying presentation of photopigment spectra. Vision Res 26, 1543-1556.

Mansfield, R.J.W. 1985. Primate photopigments and cone mechanisms. In: Fein, A. \& Levine, J.S. (eds.). The Visual System, pp. 89-106. Liss, New York.

Maximov, V.V., Orlov, O.Y. \& Reuter, T. 1985. Chromatic properties of the retinal afferents in the thalamus and the tectum of the frog (Rana temporaria). Vision Res 25, 1037-1049.

NaKa, K.-I. \& Rushton, W.A.H. 1966. An attempt to analyse colour reception by electrophysiology. 7 Physiol 185, 556-586.

NiLSSON, S.E.G. 1964a. An electron microscopic classification of the retinal receptors of the leopard frog (Rana pipiens). 7 Ultrastr Res 10, 390-419.

NiLSSON, S.E.G. 1964 b. Interreceptor contacts in the retina of the frog (Rana pipiens). 7 Ultrastr Res 11, 147-165.

Orlov, O.Y. \& Kondrashov, S.L. 1978. Colourdiscriminating functions of the visual projections in the frog. In: Orlov, O.Y. (ed.). Mechanisms of Vision in Animals, pp. 135-165. Nauka, Moscow (in Russian).

Partridge, J.C. \& De GriP, W.J. 1991. A new template for rhodopsin (vitamin $A_{1}$ based) visual pigments. Vision Res 31, 619-630.

Perry, R.J. \& MCNaughton, P.A. 1991. Response properties of cones from the retina of the tiger salamander. 7 Physiol 433, 561-587.

REUTER, T. 1969. Visual pigments and ganglion cell activity in the retinae of tadpoles and adult frogs (Rana temporaria L.). Acta Zool Fenn 122, 1-64. 
Reuter, T. \& Virtanen, K. 1972. Border and colour coding in the retina of the frog. Nature 239, 260-263.

Reuter, T.E., White, R.H. \& WaLd, G. 1971. Rhodopsin and porphyropsin fields in the adult bullfrog retina. 7 gen Physiol 58, 351-371.

SAXÉN, L. 1954. The development of the visual cells. Ann Acad Scient Fenn A 23, 1-96.
STILES, W.S. 1949. Investigations of the scotopic and trichromatic mechanisms of vision by the twocolour threshold technique. Revue d'Optique 28, 215-237.

Wald, G., Brown, P.K. \& Sмith, P.H. 1955. Iodopsin. 7 gen Physiol 38, 623-681. 